\title{
Solar Orbiter, a High-Resolution Mission to the Sun and Inner Heliosphere
}

E. Marsch ${ }^{1}$, E. Antonucci ${ }^{2}$, P. Bochsler ${ }^{3}$, J.-L. Bougeret ${ }^{4}$, B. Fleck ${ }^{5}$, R.

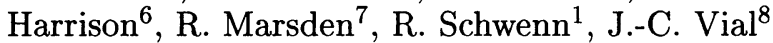

${ }^{1}$ Max-Planck-Institut für Aeronomie, Katlenburg-Lindau, Germany,

${ }^{2}$ Osservatorio Astronomico di Torino, Pino Torinese, Italy,

${ }^{3}$ Physikalisches Institut, Universität Bern, Switzerland, ${ }^{4}$ Observatoire de Paris, Meudon, France, ${ }^{5}$ ESA/GSFC, Greenbelt, Maryland, USA, ${ }^{6}$ Rutherford Appleton Laboratory, Chilton, UK, ${ }^{7}$ ESA/ESTEC, Noordwijk, The Netherlands, ${ }^{8}$ Institut d'Astrophysique Spatiale, CNRS-Université de Paris-Sud, France

\begin{abstract}
Solar Orbiter will provide, at very high spatial (35 km pixel size) and temporal resolution, novel observations of the solar atmosphere and unexplored inner heliosphere, which will be made in the heliosynchronous segments of the orbits at heliocentric distances near 45 solar radii and out of the ecliptic plane at high heliographic latitudes up to $38^{\circ}$. The Solar Orbiter will achieve its wide-ranging scientific aims with a suite of sophisticated instruments through an innovative orbit design.
\end{abstract}

\section{Scientific rationale and mission objectives}

The Sun's atmosphere and the heliosphere represent uniquely accessible domains of space, where fundamental physical processes common to solar, astrophysical and laboratory plasmas can be studied in detail and under conditions impossible to reproduce on Earth or to study from astronomical distances.

The Solar Orbiter will, through a novel orbital design, for the first time

- explore the innermost regions of our solar system,

- study the Sun from close-up (at 45 solar radii, or $0.21 \mathrm{AU}$, a $35 \mathrm{~km}$ pixel size is equivalent to 0.05 arcsec from Earth),

- fly by the Sun, tuned to its rotation, and examine the solar surface and the space above from a co-rotating vantage point,

- provide images of the polar regions from heliographic latitudes up to $38^{\circ}$.

The main scientific goals of the Solar Orbiter are

- to determine in-situ the properties and dynamics of plasma, fields and particles in the near-Sun heliosphere,

- to investigate the fine-scale structure and dynamics of the Sun's magnetised atmosphere, using close-up, high-resolution remote sensing,

- to identify the links between activity on the Sun's surface and the resulting evolution of the corona and inner heliosphere, using solar co-rotation passes, - to observe and fully characterise the Sun's polar regions and equatorial corona from high latitudes. 


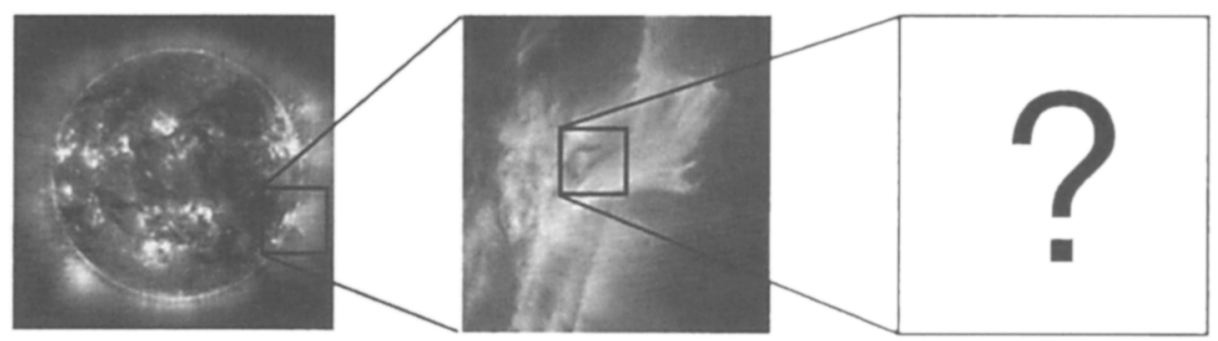

Figure 1. Orbit design of Solar Orbiter: (a) trajectory projection on the ecliptic plane; perihelion radius (b) and heliographic latitude (c) versus mission flight time in days.

The underlying basic questions relevant to astrophysics in general are: Why does the Sun vary and how does the solar dynamo work? What are the fundamental physical processes at work in the solar atmosphere and in the heliosphere? What are the links between the magnetic-field-dominated regime in the solar corona and the particle-dominated regime in the heliosphere?

\section{Four novel research perspectives}

\subsection{The Sun's magnetised plasma: Close-up observations of the so- lar atmosphere}

The close-up, high-resolution imaging of the solar atmosphere will represent a major step forward in understanding the Sun, by providing an order of magnitude improvement in spatial resolution over past missions as illustrated in Figure 1. The solar instruments will, in concert, enable us to analyse thoroughly the time-variability, evolution and fine-scale structure of the dynamic chromosphere, transition region and corona, to study the Sun's magnetic activity on multiple scales, to investigate energetic particle acceleration, confinement and release, and to reveal plasma and radiation processes underlying the heating of the corona.

\subsection{Linking the photosphere and corona to the heliosphere: Co- rotation observations}

Studies of the structure and evolution of solar features (such as sunspots, active regions, loops, prominences, streamers and coronal holes) and of their heliospheric effects are greatly complicated by the fact that the Sun rotates, and that the evolution time scales are often comparable to the rotation period. In this way, the apparent changes are entangled with such effects as centre-to-limb variation, foreshortening and projection. In order to avoid these disturbing effects it is necessary to co-rotate with the Sun. The Solar Orbiter will for the first time provide such an opportunity and thus help in resolving old and otherwise intractable problems related to the Sun's magnetic activity and the solar dynamo, e.g. the field diffusion across the solar surface. 


\subsection{Particles and fields: In-situ measurements in the inner helio- sphere}

The ultimate origins of the solar wind and heliospheric phenomena are to be searched in the dynamic corona itself. According to the SOHO findings, coronal expansion arises because of the high ion temperatures in the corona, whereas the electric field, i.e. the electron pressure gradient, and heat conduction play a minor role. The closer a spacecraft comes to the Sun, the better can the links (effects and causes) between interplanetary and solar processes, occurring on a broad range of scales in space and time, be analysed. The Solar Orbiter, while approaching the Sun to about $0.21 \mathrm{AU}$, will carry in-situ instruments with high temporal resolution, thus offering unique possibilities for resolving the plasma microstate and particle-wave interactions at their intrinsic kinetic scales.

\subsection{The Sun's polar regions and equatorial corona: Excursion out of the ecliptic}

Progress in understanding the solar dynamo will depend on how well we understand differential rotation and the circumpolar and meridional flows near the poles of the Sun. Torsional oscillations associated with the magnetically-active latitudes seem to originate close to the polar regions and then propagate toward the equator. The poles appear to rotate comparatively slowly, but the polar vortex is still not well characterised due to the serious limitations of in-ecliptic observations. The Solar Orbiter will provide the first opportunity to measure directly the magnetic field at the poles. The internal structure and dynamics of the near polar regions of the Sun is of paramount importance, and perhaps the key to our understanding of the solar activity cycle.

Table 1. Solar Remote-Sensing Instrumentation

\begin{tabular}{|c|c|c|c|c|c|}
\hline Name & Specifications & $\begin{array}{c}\text { Mass } \\
\mathrm{kg}\end{array}$ & $\begin{array}{c}\text { Size } \\
\mathrm{cm}^{3}\end{array}$ & $\begin{array}{c}\text { Power } \\
\mathrm{W}\end{array}$ & $\begin{array}{c}\text { Rate } \\
\mathrm{kb} / \mathrm{s}\end{array}$ \\
\hline $\begin{array}{c}\text { Visible-light Imager } \\
\text { and Magnetograph }\end{array}$ & Fe 630 nm line & 26 & $30 \times 40 \times 120$ & 25 & 20 \\
\hline $\begin{array}{c}\text { EUV Imager and } \\
\text { Spectrometer }\end{array}$ & $\begin{array}{c}\text { EUV emission } \\
\text { lines }\end{array}$ & 22 & $3 \times 15 \times 140$ & 25 & 17 \\
\hline EUV Imager & $\begin{array}{c}\text { He and Fe } \\
\text { Ion lines }\end{array}$ & 36 & $40 \times 40 \times 250$ & 20 & 20 \\
\hline $\begin{array}{c}\text { Ultraviolet/Visible } \\
\text { Light Coronagraph }\end{array}$ & $\begin{array}{c}\text { Coated mirror } \\
\text { coronagraph }\end{array}$ & 17 & $20 \times 20 \times 50$ & 25 & 5 \\
\hline Radiometer & Visible light & 4 & $11 \times 11 \times 22$ & 6.5 & 0.5 \\
\hline
\end{tabular}

\section{Scientific payload}

The Solar Orbiter will achieve its wide-ranging aims with a suite of sophisticated instruments, optimised to meet the science objectives. It will go to smaller distances from the Sun than any other S/C before. Therefore, the thermal load of almost 25 solar constants at perihelion presents an unprecedented technical challenge. The instruments have to be designed accordingly and comply with the 
general requirements of a low-mass, compact and integrated design, make use of on-board data compression/storage and require a modest data transmission rate. The selected strawman payload encompasses a set of remote-sensing solar instruments (see Table 1) and the in-situ heliospheric instruments (see Table 2).

Table 2. Heliospheric In-Situ Instrumentation

\begin{tabular}{|c|c|c|c|c|c|}
\hline Name & Specifications & $\begin{array}{c}\text { Mass } \\
\mathrm{kg}\end{array}$ & $\begin{array}{c}\text { Size } \\
\mathrm{cm}^{3}\end{array}$ & $\begin{array}{c}\text { Power } \\
\mathrm{W}\end{array}$ & $\begin{array}{c}\text { Rate } \\
\mathrm{kb} / \mathrm{s}\end{array}$ \\
\hline $\begin{array}{c}\text { Solar Wind } \\
\text { Plasma Analyser }\end{array}$ & $\begin{array}{c}0-30 \mathrm{keV} / \mathrm{Q} \\
0-10 \mathrm{keV}\end{array}$ & 6 & $20 \times 20 \times 20$ & 5 & 5 \\
\hline $\begin{array}{c}\text { Radio and Plasma } \\
\text { Wave Analyser }\end{array}$ & $\begin{array}{c}\mathrm{mV} / \mathrm{m}-\mathrm{V} / \mathrm{m} \\
0.1 \mathrm{nT}-\mathrm{mT}\end{array}$ & 10 & $\begin{array}{c}15 \times 20 \times 30 \\
(\text { electronics })\end{array}$ & 7.5 & 5 \\
\hline Radio Sounding & $\begin{array}{c}\mathrm{X}-\mathrm{band} \\
\mathrm{Ka}-\mathrm{band}\end{array}$ & 0.2 & $5 \times 5 \times 5$ & 3 & 0 \\
\hline $\begin{array}{c}\text { Magnetometer } \\
\text { up to } 500 \mathrm{~Hz}\end{array}$ & 1 & $10 \times 10 \times 10$ & 1 & 0.2 \\
\hline $\begin{array}{c}\text { Energetic Particle } \\
\text { Detector }\end{array}$ & $\begin{array}{c}\text { Ions/Electrons } \\
(0.01-10) \mathrm{MeV}\end{array}$ & 4 & $10 \times 20 \times 20$ & 3 & 1.8 \\
\hline $\begin{array}{c}\text { Dust Detector } \\
\text { Mass (g): } \\
10^{-16}-10^{-6}\end{array}$ & 1 & $10 \times 10 \times 10$ & 1 & 0.05 \\
\hline $\begin{array}{c}\text { Neutral Particle } \\
\text { Detector }\end{array}$ & $(0.6-100) \mathrm{keV}$ & 1 & $10 \times 10 \times 20$ & 2 & 0.3 \\
\hline Neutron detector & energy $>1 \mathrm{MeV}$ & 2 & $10 \times 10 \times 10$ & 1 & 0.15 \\
\hline
\end{tabular}

\section{Spacecraft design and mission profile}

The Solar Orbiter spacecraft (S/C) benefits from technology developed for ESA's Cornerstone mission to Mercury, BepiColombo. The S/C will be 3-axis stabilised and always Sun-pointed. Given the extreme thermal conditions (up to 25 solar constants), the thermal design of the $\mathrm{S} / \mathrm{C}$ has been considered in detail, and viable solutions have been identified. The orbit design is based on solar electric propulsion (SEP) in conjunction with multiple planetary swing-by manoeuvres.

In order to be compliant with mission requirements and $\mathrm{S} / \mathrm{C}$ needs, it is necessary to carry two sets of solar arrays on the Solar Orbiter. The first set will be used only during the cruise phase for propulsion and jettisoned after the last firing. The second set is kept and protected by angling it away from the Sun during the perihelion passages. The payload mass is $130 \mathrm{~kg}$. The total S/C mass of the Solar Orbiter is $1296 \mathrm{~kg}$, compatible with a Soyuz-Fregat launch from Baikonur.

The mission profile (see Figure 2) uses an interleaving of Earth/Venus gravity assists and SEP firings and is composed of three phases:

- The cruise phase, starting at spacecraft separation from the launcher, and ending at the start of scientific operations.

- The nominal mission phase, during which the main scientific measurements will be performed.

- The extended mission phase, when further gravity assist manoeuvres will allow higher inclination observations. 

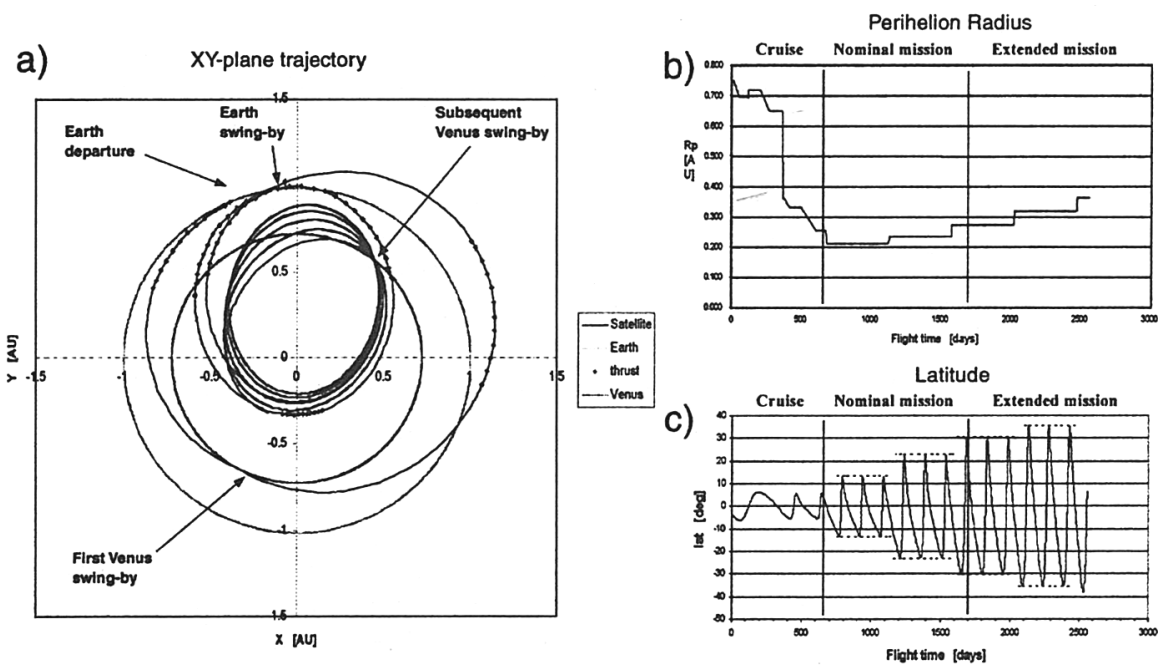

Figure 2. Orbit design of Solar Orbiter: (a) trajectory projection on the ecliptic plane; perihelion radius (b) and heliographic latitude (c) versus mission flight time in days.

It will take the Solar Orbiter only two years to reach a perihelion of 45 solar radii at an orbital period of 149 days. Within the nominal 5-year mission phase, the Solar Orbiter will perform several swing-by manoeuvres at Venus, in order to increase the inclination of the orbital plane to $30^{\circ}$ with respect to the solar equator. During an extended mission phase of about two years the inclination will be further increased to $38^{\circ}$. The selected orbit assures the observation of the Sun from close-up and at scientifically satisfactory heliographic latitudes.

The Solar Orbiter telemetry will be handled via X-band low-gain antennae and by a 2-axis steerable Ka-band high-gain antenna. The mission will have variable transmission phases, the durations of which vary with distance from Earth. Three scientific observation periods (each ten days long) are considered per S/C revolution about the Sun, with the strategy that these periods are centred on the passages through maximum southern and northern latitude and perihelion. We anticipate a high-data-rate acquisition of $75 \mathrm{~kb} / \mathrm{s}$ in the nominal observation mode. In order to avoid antenna pattern distorsion, a minimum Sun-S/C distance of $0.5 \mathrm{AU}$ has been fixed for downloading.

\section{References}

Marsch, E. \& al. 2000, Solar Orbiter, High-resolution Mission to the Sun and Inner Heliosphere, A mission proposal submitted in response to the ESA call for flexible mission ideas, 27 January

Pre-Assessment Study Report Solar Orbiter, ESA CDF-02(A), October 1999

Solar Orbiter - A High Resolution Mission to the Sun and Inner Heliosphere, Assessment Study Report, ESA SCI (2000) 6, July 2000 